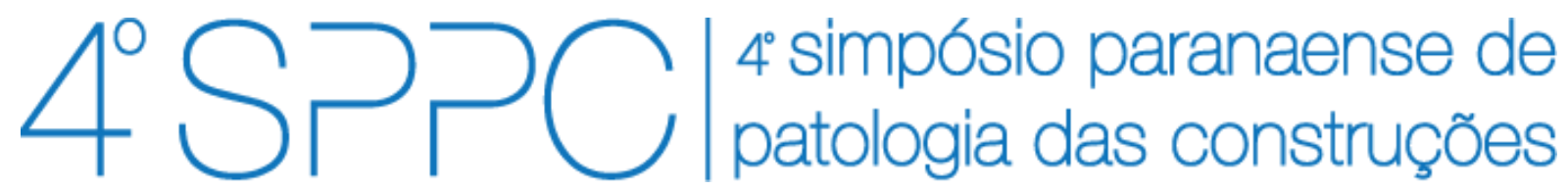

ISSN 2526-7248 artigo n. 4SPPC153, pp. 482-490, 2019

\title{
Análise do comportamento estrutural de diferentes métodos de recuperação parcial de estribos em pilares de concreto armado em escala reduzida
}

\author{
Deprá, Monalisa Tainá Finger ${ }^{1}$; Sagave, André Matte ${ }^{2}$ \\ ${ }^{1}$ Monalisa Tainá Finger Deprá, Universidade do Vale do Itajaí, mona_taina@hotmail.com \\ ${ }^{2}$ André Matte Sagave, Universidade do Vale do Itajaí, sagave@univali.com.br
}

\begin{abstract}
Resumo: As primeiras armaduras afetadas pela corrosão, tendem a ser os estribos, pois localizam-se próximo à face do elemento. Em pilares de fachada em atmosferas litorâneas, as faces não ficam expostas à mesma intensidade de agressão dos agentes causadores da corrosão. Assim, quando somente algumas das faces estiverem danificadas, este estudo propõe possíveis técnicas de recuperação parcial de estribos em pilares. Foram produzidos 28 pilares de concreto armado em escala reduzida com seção transversal de $10 \times 20 \mathrm{~cm}$, e altura de $65 \mathrm{~cm}$, nestes, foram executados cinco modelos de recuperação. Foram moldados 4 pilares de referência e para cada modelo de recuperação foram moldados, no mínimo, 4 pilares para cada modelo. Nos pilares de recuperação foi simulado a perda de seção nos três estribos mais superiores e inferiores, em seguida realizado o processo de recuperação e posteriormente foram ensaiados à compressão axial, parte deles com um dia de idade da recuperação e o restante com quatorze dias. Foi avaliado a resistência dos pilares e os danos às armaduras, analisando assim o comportamento geral de cada modelo de recuperação. Traçando um comparativo entre os modelos e os pilares de referência pode concluir-se que os modelos sem gancho e com gancho reto obtiveram os piores desempenhos. Por outro lado, o modelo com gancho inclinado obteve o melhor comportamento em relação aos demais.
\end{abstract}

Palavras-chave: Corrosão, Estribos, Pilares, Recuperação estrutural.

Abstract: The first armor affected by corrosion, tend to be the stirrups, because they are located close to the face of the element. On façade pillars in coastal atmospheres, the faces are not exposed to the same intensity of aggression as the causers of corrosion. Thus, when only some of the faces are damaged, this study proposes possible techniques of partial recovery of STIRRUPMS in pillars. We produced 28 pillars of reinforced concrete with a transverse section of $10 \times 20 \mathrm{~cm}$, and height of $65 \mathrm{~cm}$, in these, five recovery models were performed. Four reference pillars were molded and for each recovery model, at least 4 pillars were molded for each model. In the recovery pillars was simulated the loss of section in the three upper and lower Stirrupes, then performed the recovery process and subsequently were tested to axial compression, part of them with one day of age of recovery and the remainder with Fourteen days. The resistance of the pillars and the damage to the armor were evaluated, thus analyzing the overall behavior of each recovery model. By tracing a comparison between the models and the reference pillars it can be concluded that the models without hook and with straight hook obtained the worst performances. On the other hand, the model with inclined hook obtained the best behavior in relation to the others.

Keywords: Corrosion, Stirrups, Pillars, Structural Rehabilitation. 
DEPRÁ, M. T. F; SAGAVE, A. M. ANÁLISE DO COMPORTAMENTO ESTRUTURAL DE DIFERENTES MÉTODOS DE RECUPERAÇÃO DE ESTRIBOS EM PILARES DE CONCRETO ARMADO EM ESCALA REDUZIDA. $4^{\circ}$ Simpósio Paranaense de Patologia das Construções (40 SPPC), artigo 4SPPC153, pp. 482 - 490, 2019. DOI: 10.4322/2526-7248.070

\section{Introdução}

Por muito tempo vêm sendo consolidado, mundialmente, o conceito de que as estruturas de concreto armado são eternas. Contudo, os tempos modernos ditaram a certeza de que o concreto, como material de construção, é instável ao longo do tempo, alterando suas propriedades físicas e químicas em função da característica de seus componentes e das respostas destes às condicionantes do meio ambiente (SOUZA; RIPPER [1]). É frequente deparar-se com diversas situações em que edificações exteriorizam os mais diversos defeitos, sejam eles acarretados por falhas humanas, de execução ou manutenção.

O estudo das patologias das construções emerge, segundo Helene [2], com o intuito de estudar os sintomas, os mecanismos, as causas e as origens dos defeitos das construções civis, ou seja, é o estudo das partes que compõem o diagnóstico do problema.

A corrosão é um dos principais fatores responsáveis pela deterioração do aço em estruturas de concreto armado, e está constantemente transformando os materiais metálicos de modo que a durabilidade e desempenho dos mesmos deixam de satisfazer os fins a que se destinam podendo causar sérios danos na estrutura e até levá-la ao colapso (GENTIL [3]).

Quando se trata de corrosão, as primeiras armaduras a serem afetadas, tanto em pilares como em vigas, tendem a ser os estribos, pois localizam-se mais próximo à face do elemento possuindo menor cobrimento de proteção se comparado às armaduras longitudinais.

Em pilares, geralmente as faces não ficam expostas à mesma intensidade de agressão dos agentes causadores da corrosão, como é o caso de pilares de fachada em atmosferas litorâneas que ficam com uma ou duas faces expostas e as demais protegidas pelo ambiente interno ou em função da execução de paredes. Assim, segundo Rebello [4] a reposição parcial dos estribos pode ser a solução adotada para o trabalho de recuperação, em virtude de, ocasionalmente, somente algumas das faces estarem danificadas.

Não foram identificados em referencial bibliográfico, procedimentos recomendados, de forma direta e específica, para a recomposição parcial dos estribos. Diante desse contexto, inclui-se o presente estudo com a finalidade de avaliar o comportamento estrutural de diferentes métodos de recuperação parcial de estribos em pilares de concreto armado.

\section{Metodologia}

\subsection{Procedimentos e instrumentos de coleta e análise de informações}

O presente trabalho trata-se de uma continuação do trabalho de conclusão de curso de Rebello [4]. Nos modelos desenvolvidos pelo autor, o procedimento de recuperação dos estribos foi realizado na região central dos pilares, porém as rupturas durante os ensaios, ocorreram próximo às extremidades. Buscando ampliar a discussão do trabalho e, dando continuidade ao mesmo, desenvolveu-se agora a recuperação dos estribos nas extremidades dos pilares. 
DEPRÁ, M. T. F; SAGAVE, A. M. ANÁLISE DO COMPORTAMENTO ESTRUTURAL DE DIFERENTES MÉTODOS DE RECUPERAÇÃO DE ESTRIBOS EM PILARES DE CONCRETO ARMADO EM ESCALA REDUZIDA. $4^{\circ}$ Simpósio Paranaense de Patologia das Construções (40 SPPC), artigo 4SPPC153, pp. 482 - 490, 2019. DOI: 10.4322/2526-7248.070

Assim, tomou-se como base o traço referência utilizado por Rebello [4], expresso na Tabela 1, almejando uma resistência caraterística (fck) de 30MPa aos 28 dias e um consumo de cimento de $330,00 \mathrm{~kg} / \mathrm{m}^{3}$ contando com a utilização de um aditivo superplastificante.

Tabela 1: Traço Referência

\begin{tabular}{cccc}
\hline Cimento & Areia & Brita & Água \\
\hline 1 & 1,74 & 2,41 & 0,55 \\
\hline
\end{tabular}

\subsubsection{Dimensionamento e produção dos pilares}

Para o dimensionamento dos pilares, manteve-se as dimensões dos pilares do estudo anterior, sendo a seção transversal de $10 \mathrm{~cm} \times 20 \mathrm{~cm}$ e altura de $65 \mathrm{~cm}$. A altura do pilar especificamente foi definida em função da limitação imposta pela prensa hidráulica disponível no Laboratório de Pesquisa Tecnológica em Engenharia Civil (LATEC). Sendo assim, algumas considerações da NBR 6118:2014 [5] acerca da geometria de pilares, não puderam ser atendidas.

De acordo com Rebello [4], se tratando de um estudo comparativo, mantendo-se a relação de seção e altura das peças ensaiadas, é possível traçar um comportamento que pode ser extrapolado para peças de maiores dimensões.

Para determinação da área de aço a ser utilizada nos pilares, a condição prescrita na NBR 6118:2014 [5] que limita a utilização de bitola mínima para armaduras longitudinais de $\phi 10,00 \mathrm{~mm}$ não pode ser seguida, porém, como se trata de um estudo comparativo, a adoção de bitolas menores interferem em igualdade de condições em todos os ensaios e, portanto, não se enquadra como uma variável a ser interpretada nos resultados dos ensaios.

Assim, as barras longitudinais às quais fariam maior concordância com a seção do pilar e que supririam a armadura mínima de aço, para um concreto com fck de 30 MPa, o pmín adotado é de $0,15 \%$, totalizando uma área mínima de aço de $0,30 \mathrm{~cm}^{2}$, determinando-se assim, a utilização de 4 barras longitudinais de bitola de CA-50 de $\phi 8,00 \mathrm{~mm}$.

Já para a determinação das armaduras transversais, conforme preconiza a NBR 6118:2014 [5], foi adotado o espaçamento máximo de St $\leq 12 \phi$, resultando em um espaçamento máximo de $9,60 \mathrm{~cm}$, adotando-se $8,50 \mathrm{~cm}$ para os espaçamentos internos. Com o valor de área mínima obtida, determinou-se a utilização de bitola de CA-60 de $\phi 5,00 \mathrm{~mm}$ para os estribos. Para simetria entre os espaçamentos, deixouse uma folga de $2,75 \mathrm{~cm}$ em cada ponta do pilar, a fim de evitar qualquer dano ou má fixação do estribo. O detalhamento final pode ser observado nas Figuras 1 e 2 .

Foi utilizada para produção, seis formas de pilares. Em seguida foram montadas as armaduras dos pilares, colocadas nas formas e concretadas. Para o modelo de referência, foram moldados 4 pilares e, para cada modelo de recuperação (5 modelos), foram moldados 4 pilares, totalizando 24 pilares. Ainda, foram moldados 4 pilares-reserva para eventuais necessidades de análises. 


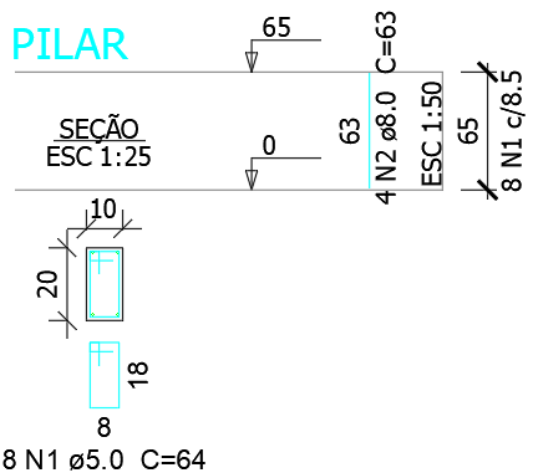

Figura 1: Detalhamento dos pilares

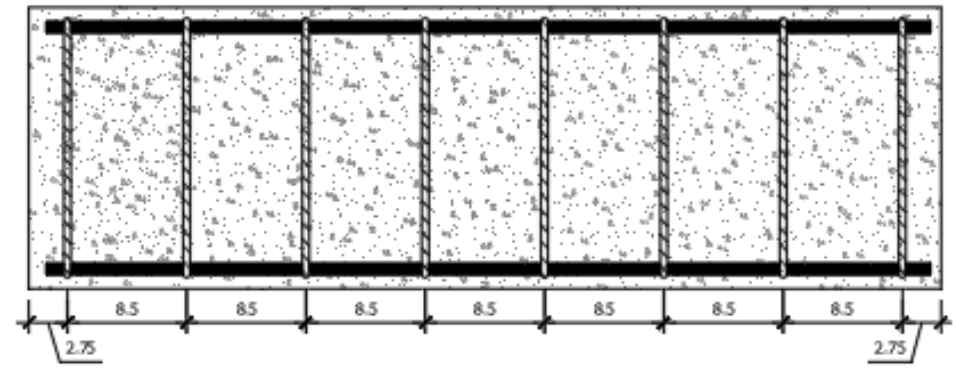

Figura 2: Modelo de armação dos pilares

Os pilares foram adensados manualmente, deitados na sua maior dimensão da seção transversal $(20 \mathrm{~cm})$ com a finalidade de facilitar o adensamento e as etapas seguintes de recuperação.

Os pilares de referência tiveram sua seção integralmente concretada, e os modelos de recuperação foram concretados parcialmente conforme Figura 3. Todos foram desmoldados após passado 24 horas da concretagem e armazenados em câmara úmida até a data da recuperação.

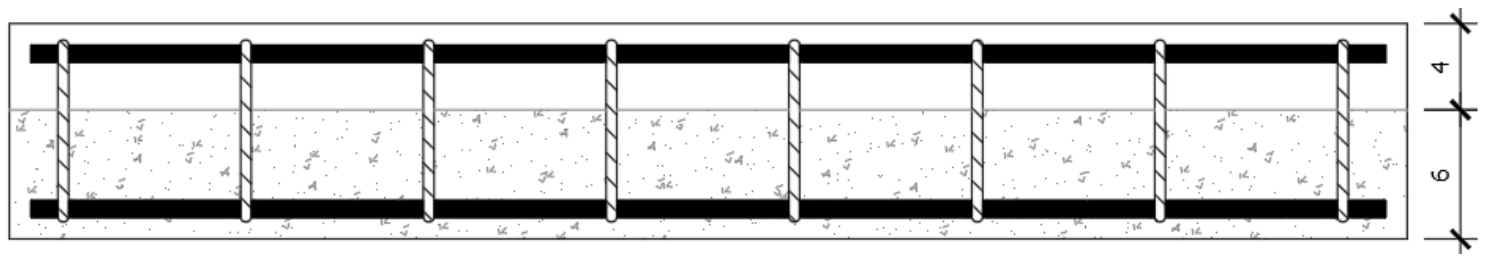

Figura 3: Concretagem parcial dos modelos de recuperação

Todo concreto foi produzido em cinco dias, em função do número de formas disponíveis para confecção dos pilares. Para cada dia de produção foi realizado um amassamento. Seguindo o que consta na NBR 5738:2015 [6], para cada amassamento foram moldados dois corpos-de prova com $10 \mathrm{~cm}$ de diâmetro e $20 \mathrm{~cm}$ de altura, para aferir posteriormente a resistência do concreto produzido.

Dos 4 pilares de cada modelo de recuperação, dois deles passaram pelo processo de recuperação aos 14 dias de idade do concreto e os demais, aos 27 dias. 


\subsubsection{Processo de recuperação dos estribos}

Após a cura prevista para os modelos de recuperação, foi realizado o corte dos estribos afim de simular a perda de seção da armadura transversal. Foi realizado o corte dos três estribos mais superiores e mais inferiores em relação à altura do pilar. A extensão da perda de seção foi fixada em $8,5 \mathrm{~cm}$ (Figura 4), pois para o processo de recuperação do Modelo 5 , exige um comprimento de solda de $4 \mathrm{~cm}$ em cada extremidade do estribo.
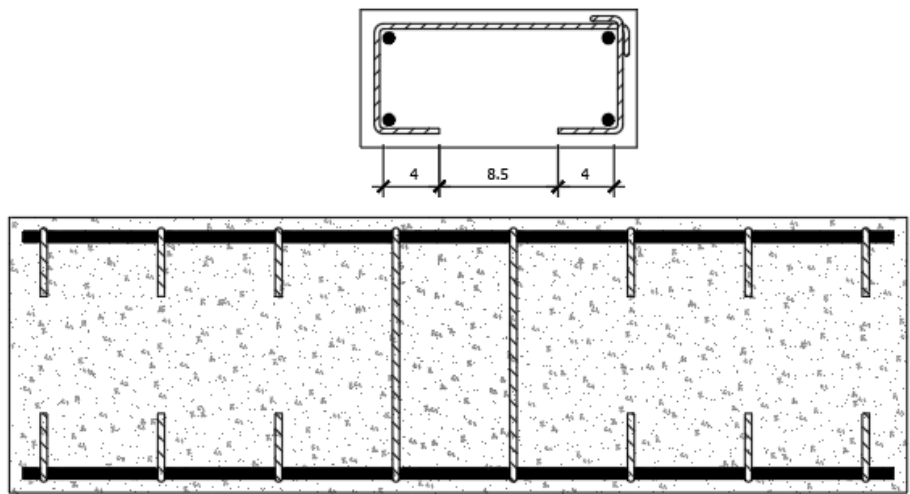

Figura 4: Seccionamento dos estribos

Seguiu-se uma sequência de passos para o processo de recuperação, iniciando pelo lixamento da base de aderência do graute para criar uma superfície rugosa, em seguida a lavagem do pilar para remoção de impurezas resultantes do lixamento, que podem prejudicar a aderência do graute ao concreto em seguida a secagem do pilar ao ar livre, e por fim, a recomposição dos estribos danificados de acordo com as tipologias demonstradas na Figura 5.
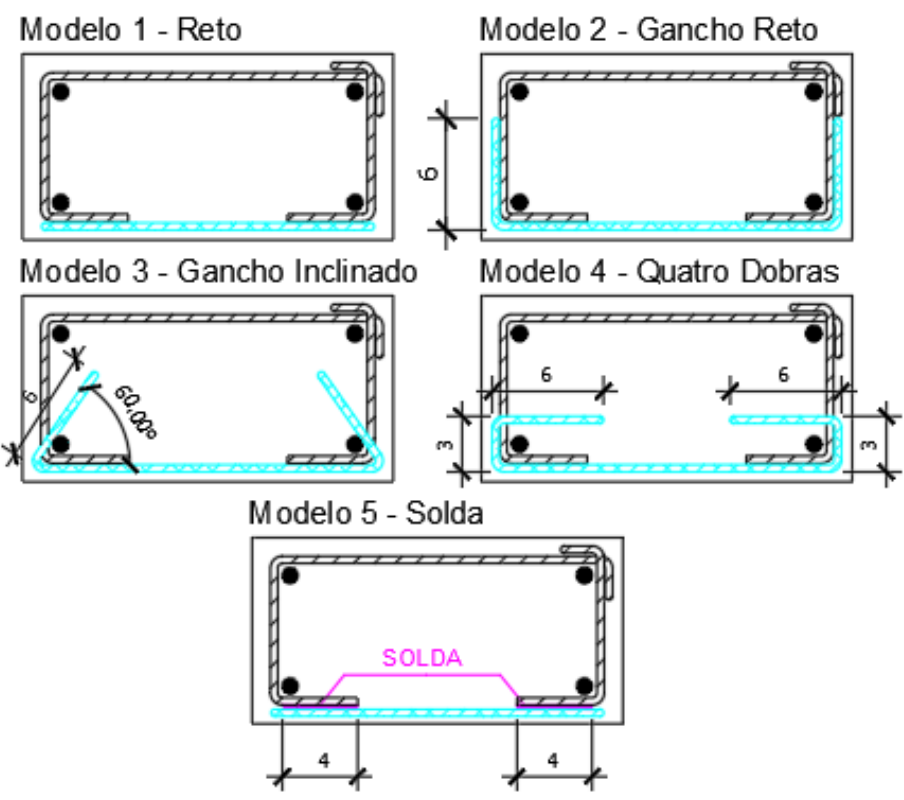

Figura 5: Modelos de Recuperação 
DEPRÁ, M. T. F; SAGAVE, A. M. ANÁLISE DO COMPORTAMENTO ESTRUTURAL DE DIFERENTES MÉTODOS DE RECUPERAÇÃO DE ESTRIBOS EM PILARES DE CONCRETO ARMADO EM ESCALA REDUZIDA. $4^{\circ}$ Simpósio Paranaense de Patologia das Construções (40 SPPC), artigo 4SPPC153, pp. 482 - 490, 2019. DOI: 10.4322/2526-7248.070

Após a conclusão do processo de recuperação, foi feita a recomposição da seção com graute, e os pilares foram novamente armazenados em câmara úmida, desmoldados ao passar 24 horas da recuperação, e ali permaneceram até a data prevista de rompimento. Os pilares que foram rompidos com um dia de idade do graute, foram desmoldados pouco antes do rompimento.

\subsubsection{Ensaio dos Pilares}

Anteriormente à execução do ensaio foi necessário preparar os pilares. O processo consistiu na lixação da base e do topo do pilar, a fim de remover irregularidades de forma, evitando assim uma concentração de tensões que poderiam prejudicar as análises dos resultados. Ainda, foi necessário demarcar os eixos centrais dos pilares e da prensa hidráulica para evitar excentricidades e consequentemente momentos indesejados, garantindo assim uma distribuição de carga uniforme.

Todos os pilares foram ensaiados à compressão axial com o auxílio de uma prensa hidráulica com capacidade de carga de 100 toneladas, que aplica uma carga de forma concentrada sobre o pilar. Nos ensaios, além da resistência final obtida, também foram analisados o tipo e o local da ruptura, identificando possíveis interferência dos estribos de recuperação.

\section{Resultados e Análises}

\subsection{Concreto}

Todos os corpos-de-prova foram ensaiados à compressão axial, apresentando resistêcias que atenderam o especificado no traço de 30MPa. Somente os corpos-deprova moldados em um dia específico apresentaram resistência abaixo de 30MPa, não sendo encontrada uma justificativa para essa redução que ficou em média 27Mpa. Esse fator foi considerado nas análises dos modelos produzidos nesta data.

\subsection{Pilares}

Em relação à resistência dos pilares, foi realizada uma análise estatística através do método ANOVA, e evidenciado que as pequenas diferenças de resistência encontradas, se analisadas estatisticamente, não diferem significativamente, inferindo assim em igualdade de condições de resistência entre os modelos.

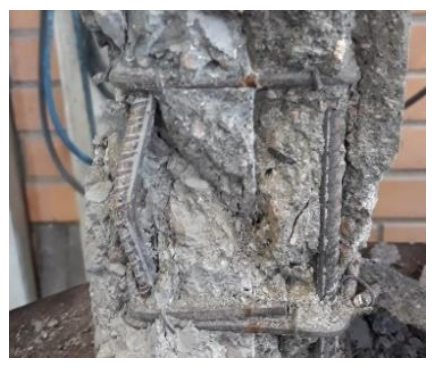

Figura 6: Modelo de Recuperação 1 - Reto 


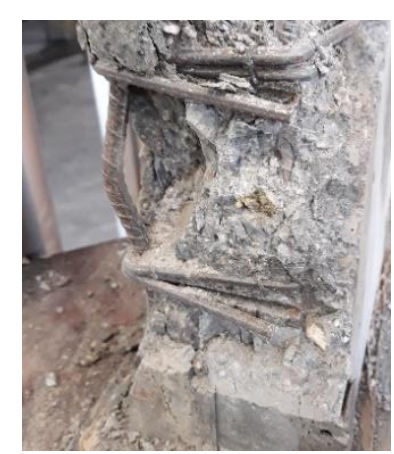

Figura 7: Modelo de recuperação 2 Gancho Reto

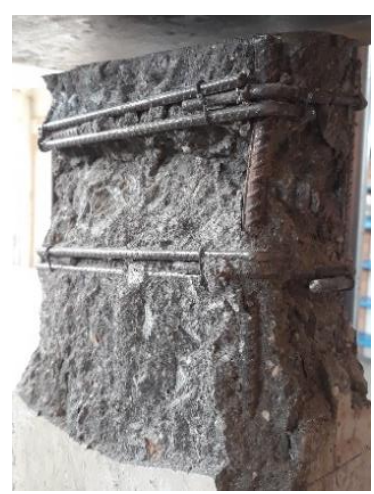

Figura 9: Modelo de recuperação 4 Quatro dobras

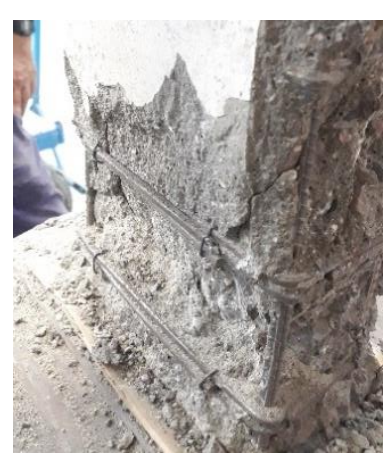

Figura 8: Modelo de recuperação 3 Gancho inclinado

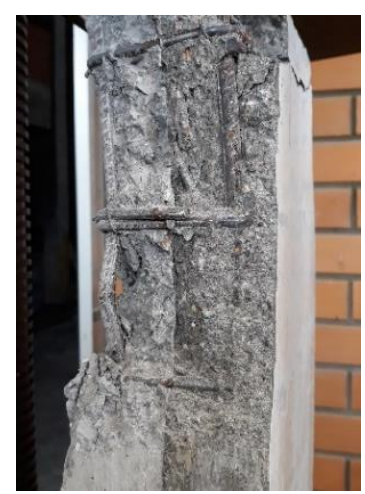

Figura 10: Modelo de recuperação 5 Solda

\subsection{Danos às armaduras longitudinais}

Com relação aos danos às armaduras longitudinais, verificou-se a ocorrência de flambagem no topo e na base do pilar, geralmente entre o segundo e terceiro estribo. Esse comportamento pode ser reflexo do formato do pilar, que por se tratar de um pilar curto, os maiores esforços concentram-se nestas regiões.

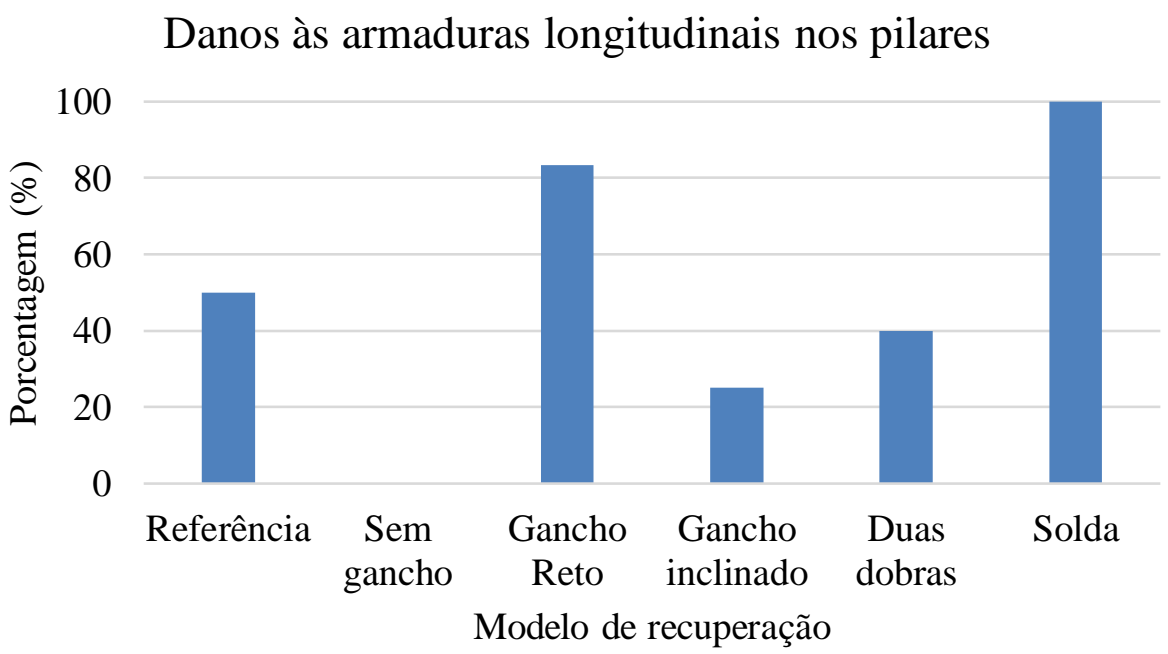

Gráfico 1: Danos às armaduras longitudinais 
DEPRÁ, M. T. F; SAGAVE, A. M. ANÁLISE DO COMPORTAMENTO ESTRUTURAL DE DIFERENTES MÉTODOS DE RECUPERAÇÃO DE ESTRIBOS EM PILARES DE CONCRETO ARMADO EM ESCALA REDUZIDA. $4^{\circ}$ Simpósio Paranaense de Patologia das Construções (40 SPPC), artigo 4SPPC153, pp. 482 - 490, 2019. DOI: 10.4322/2526-7248.070

\subsection{Danos aos estribos de recuperação}

Para a análise dos estribos de recuperação fez-se o processo de escarificação do concreto nas regiões onde observou-se a ruptura do pilar. Assim pode-se visualizar os danos e com isso traçar um comparativo da quantidade de estribos danificados entre os modelos de recuperação, como pode ser visualizado no Gráfico 2.

Danos aos estribos de recuperação nos pilares

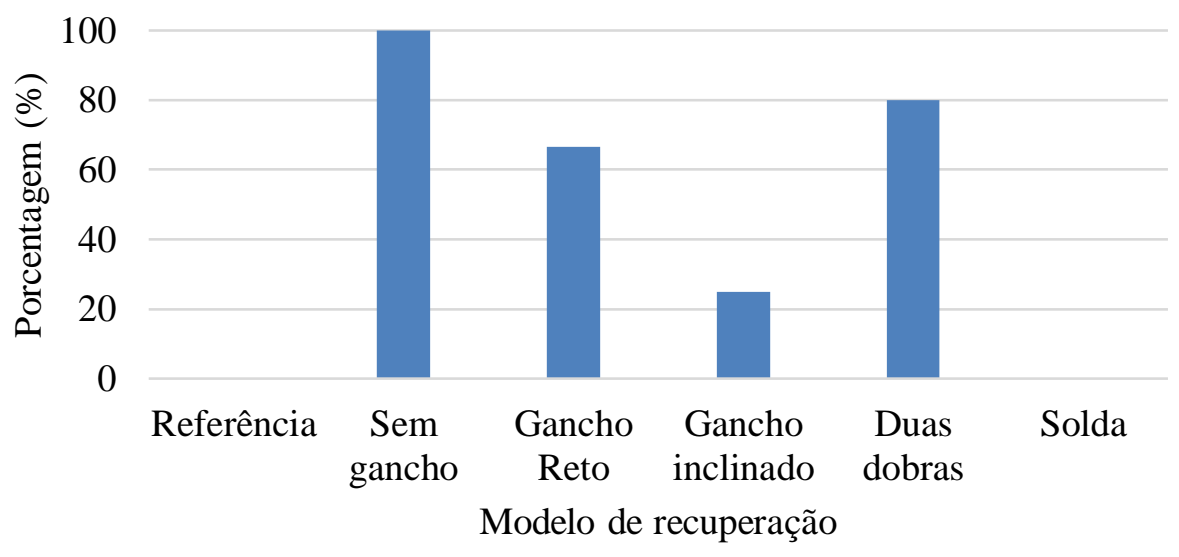

Gráfico 2: Danos aos estribos de recuperação

\section{Conclusões}

Efetuando uma análise conjunta dos diversos fatores abordados, pode se concluir que os modelos de recuperação sem gancho, com duas dobras, com gancho reto e com solda, foram os que apresentaram pior desempenho, revelando danos tanto às armaduras longitudinais quanto aos estribos originais e de recuperação.

Em linhas gerais, o modelo com gancho inclinado apresentou-se como o modelo de recuperação mais eficiente, uma vez que não apresentou danos aos estribos originais, mostrou um leve afrouxamento dos estribos de recuperação, que pode ter sido resultado da amarração do estribo, e em relação à flambagem da armadura, se evidenciou em somente um pilar, sendo na região do concreto, e não da recuperação.

\section{Referências}

[1] Souza, Vicente Custódio Moreira de; RIPPER, Thomaz. Patologia, recuperação e reforço de estruturas de concreto. São Paulo: Pini, 1998, 255p.

[2] Helene, Paulo Roberto do Lago. Manual para reparo, reforço e proteção de estruturas de concreto. 2. ed. São Paulo: Pini, 1992, 213p.

[3] Gentil, Vicente. Corrosão. 6. ed. Rio de Janeiro: Ltc, 2011. 360 p. 
DEPRÁ, M. T. F; SAGAVE, A. M. ANÁLISE DO COMPORTAMENTO ESTRUTURAL DE DIFERENTES MÉTODOS DE RECUPERAÇÃO DE ESTRIBOS EM PILARES DE CONCRETO ARMADO EM ESCALA REDUZIDA. 40 Simpósio Paranaense de Patologia das Construções (40 SPPC), artigo 4SPPC153, pp. 482 - 490, 2019. DOI: 10.4322/2526-7248.070

[4] Rebello, Matheus. Eficiência e viabilidade técnica de diferentes métodos de recuperação de estribos em pilares de concreto armado em escala reduzida. 2017. 137 f. TCC (Graduação) - Curso de Engenharia Civil, Centro de Ciências Tecnológicas da Terra e do Mar, Universidade do Vale do Itajaí, Itajaí, 2017.

[5] NBR 6118: Projetos e estruturas de concreto - Procedimento. 3 ed. Rio de Janeiro, 2014. $238 \mathrm{p}$.

[6] NBR 5738: Concreto - Procedimento para moldagem e cura de corpos-de-prova. 2 ed. Rio de Janeiro, 2015. 9 p. 